\title{
Polar 3D Transformation of the Full Gradient of Attractive Potential
}

\author{
Gennady Prostolupov, Michail Tarantin \\ Laboratory of Geopotential Fields, Perm, Russia \\ Email: gravik2@rambler.ru
}

Received December 21, 2011; revised February 9, 2012; accepted March 11, 2012

\begin{abstract}
The method of 3D polar transformation of full gravity potential gradient vectors is based on the geometric properties of the crossing points of complete gradient of the potential to localize the source region that causes the observed anomaly. The cross-points - poles - are defined for rectangular polygons of different sizes where the full gradient vector is defined at every vertex. The polygon size range could be specified. The set of poles, positive and negative, is then represented on the 3D chart in the form of clusters of dots or cubes and can be considered as a model image of the sources, intended for visual analysis and further interpretation.
\end{abstract}

Keywords: Gravity; Anomaly; Interpretation; Model; Vector; Full Gradient; 3D Chart

\section{Introduction}

Developing new methods of data express interpretation, that give the first approximation for quantity analysis and geological interpretation, is of actual type. Presented method of a polar transformation, as Euler deconvolution [1] or tensor deconvolution [2], is just a transformation of obtained data and is used primary for visual analysis. Attention to such kind of methods is due to its low cost in computing. The most close to such methods are that based on a theory of special points. But the methods mentioned above are much more productive but less strong with theory.

\section{Theory}

The method described in this paper uses the geometrical properties of the full vectors of potential gradient in three-dimensional space: they concentrate in the positive mass direction and disperse when mass is negative. The crossing point of vector-lines lies in nearby of perturbation mass and coincides with that mass if it is of pointtype. For brief these points of line crossing are called by the authors as poles, because they became a special property - sign of the attached mass (positive or negative).

All three gradients could be found from the vertical one using the method of source-point approximation [3]. For survey data the construction of point masses below every measure point is build. The gravity effect of such construction should be equal to the measured data with the given accuracy. When having such a system of masses any type of derivatives could be found. For example, the first order derivatives could be found by (1).

$$
\begin{aligned}
& V_{x}=G \iint_{x y} \sigma r^{-3}(\xi-x) \mathrm{d} x \mathrm{~d} y \\
& V_{y}=G \iint_{x y} \sigma r^{-3}(\eta-y) \mathrm{d} x \mathrm{~d} y \\
& V_{z}=G \iint_{x y} \sigma r^{-3}(\varsigma-z) \mathrm{d} x \mathrm{~d} y
\end{aligned}
$$

When having the gravity effect of a sphere the crosspoint of any lines of full gradient vectors coincides with the center of that sphere. But the real field is additive and complex and it is practically impossible to find 3 vectors of attractive force that cross in one point of a realm. Nevertheless we had introduced a following algorithm to get the coordinates of poles.

Let's consider determination of a cross-point coordinates of a single mass in a plane by one pair of vectors (Figure 1). Let us know the attractive force vectors in two points A and B. Obviously both of them would look in the direction of the attractor and exactly in its center. So we could determine the mass center position of the body by two coordinates $X_{M}$ and $Z_{M}$ by (2).

Having 2 vectors we can define 5 types of poles possible. When both of the vectors concentrate in the lower half-space we got a positive pole, when both of them concentrate in the upper part of the half-space we got a negative pole. When vectors disperse to lower half-space or disperse to upper half-space we got weakly negative or weakly positive type respectively. The last type is neutral. 


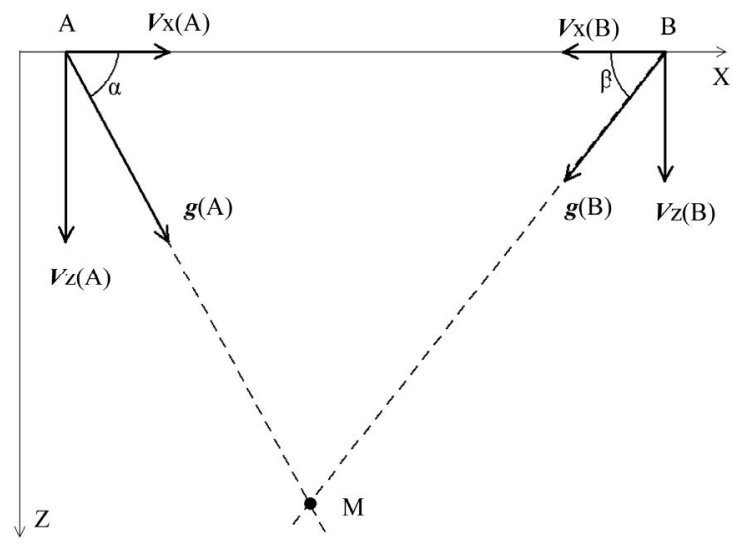

Figure 1. For cross-point coordinates definition.

$$
\left\{\begin{array}{l}
Z_{M}=\frac{X_{B}-X_{A}}{\operatorname{ctg} \alpha-\operatorname{ctg} \beta} \\
X_{M}=X_{A}+Z_{M} \cdot \operatorname{ctg} \alpha
\end{array}\right.
$$

For all calculations in three-dimensional space the 4vertex polygons are used. Every vertex is placed in the measured point or in the grid point, if the measured data had been gridded and processed. All further calculations proceed only with polygons having all vectors directed in one half-space at the same time. When all vectors point up we got a negative or weakly positive pole, when all vectors point down we got a positive or weakly negative pole, depending on convergence of vectors. None of other combinations is used at the time. Having 4 vertexes we can define 6 pairs of vectors ( 4 at sides and 2 at diagonals) to find 6 triplets of pole coordinates. The final coordinates of the pole for the polygon are the mean-averaged of all got for it. The range of polygon size variations could be set by operator. The algorithm uses all possible polygons within data grid.

As a result we got a $3 \mathrm{D}$ chart of poles $\mathrm{P}++$ and $\mathrm{P}--$. After some filtering with the chosen algorithm (for example, one from [4]) the chart is divided into set of clusters. These clusters in turn give us positions and approximate shapes of the sources of anomalies. The developed program based on this algorithm allows fieldsource bodies to be a sphere, combination of blocks or a star-body with an equivalent volume and mass. Having a full force vector the attractive mass is calculated as $m=|g| \cdot r^{2} / G$, where $G$ is the gravitational constant, $g$ is the full force vector, $r$ is a distance from the grid point to the pole point. The mass of a cluster is an average mass of all parts in it: for sphere and star-body - of all poles and for combination of blocks - of all blocks. The anomalous density of a field-source could be determined by its mass and volume.

\section{Examples}

As a theoretical example let's consider results of two point source field processing. These sources have differrent masses and lay on different depths: 0.5 and $0.75 \mathrm{~km}$ (Figure 2). Determination of the position of sources gives results that are close to real. The discrepancies of coordinates and mass lay in 2 per cent boundary. Results for 5 point-sources show the same quality of localization.

As follows from the theory of a method [5] the most appropriate type of fields to process is a point-source field. But the real observed field are too complicated and do not have such a morphology. So let's consider a non-point model - the field of a material rod. Such type of model could be used in working with mines and pits, gas pipelines or any lengthy objects. Figure 3 shows the results of three rods field processing. It is seen that the red point sources are combined in lengthy clusters of rod type.

Comparing with strong defined poles $\mathrm{P}++$ and $\mathrm{P}--$ the weakly defined poles are non-obvious or cryptic for sources. During the processing of a real field it is impossible to determine the type (positive or negative) of its sources. We can not distinguish the portions of softening of consolidated ground from the consolidation of soft soils. So the use of weakly determined poles could be informative.

If, for example, we consider a source field defined by a sine function then it could be caused either positive masses or negative. Using only strong defined poles gives us only one type of sources while weak defined poles gives both types (positive and negative). Weak defined poles for rod field case is shown in Figure 3 with blue.

The next example is the field of a lengthy L-rod. Such
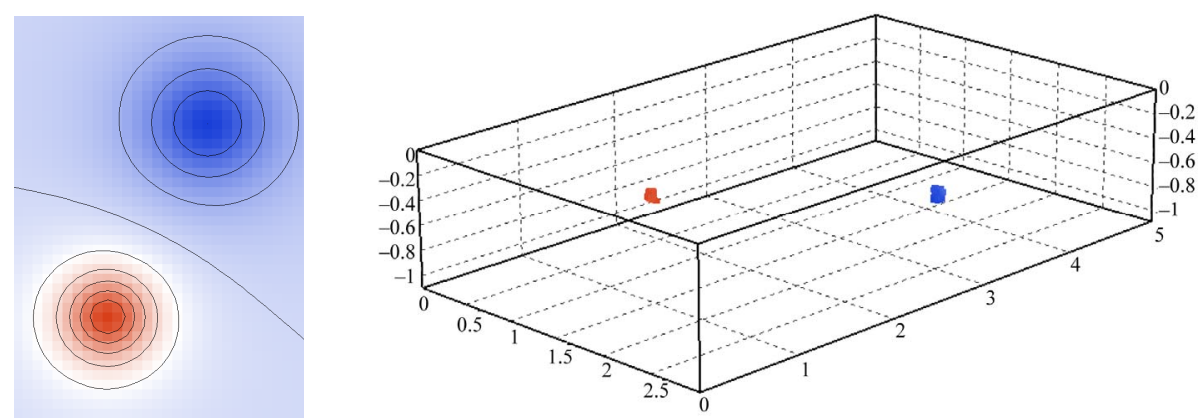

Figure 2. Model gravity field of two point mass (left) and the result of mass positions determination (right). 
kind of source could model an ore body. As shown in Figure 4 the result cluster shape is in good agreement with the source model, but the left part of it is a bit lower.

The tests show that the best result for complex fields could be gained when using the combination of divided and processed apart fields. These parted results are to be combined in one diagram like one in Figure 5. That figure shows the result of $2 \mathrm{~km}$ deep reef trap field processing. The trap and its envelope could be located on the diagram.

\section{Conclusion}

The geometrical property of an attraction potential gradient to point to the force sources allows building models of equivalent mass distribution. The difference of the shown above diagrams from the transformed field diagram or up- and down-continuous is as follows. Here we have a $3 \mathrm{D}$ view of equivalent mass source chart with definite properties (position, size and mass) in spite of 2D field being interpolated in 3D. These diagrams could be
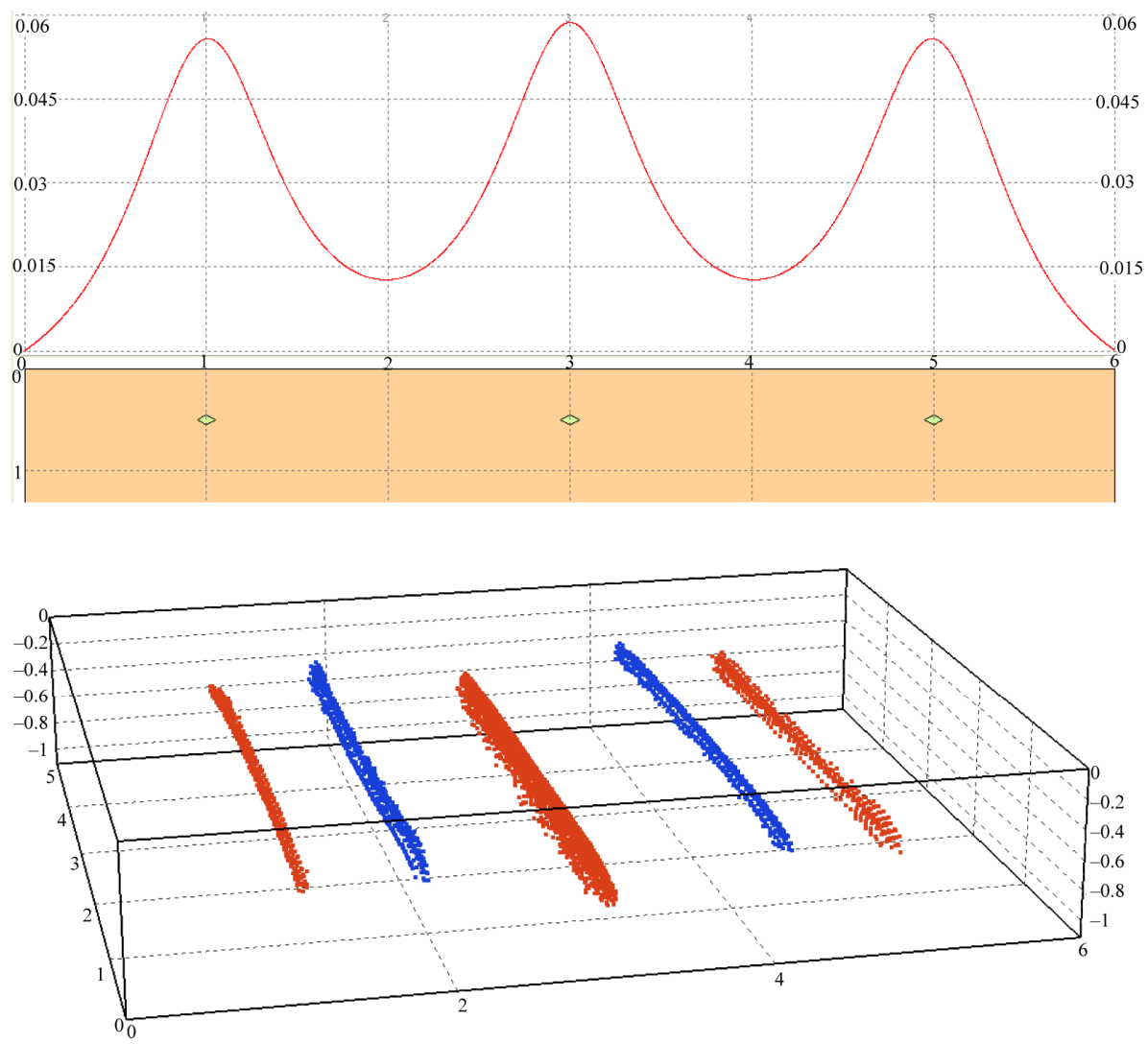

Figure 3. 2D field shape for 3 rods and the result of this field processing.
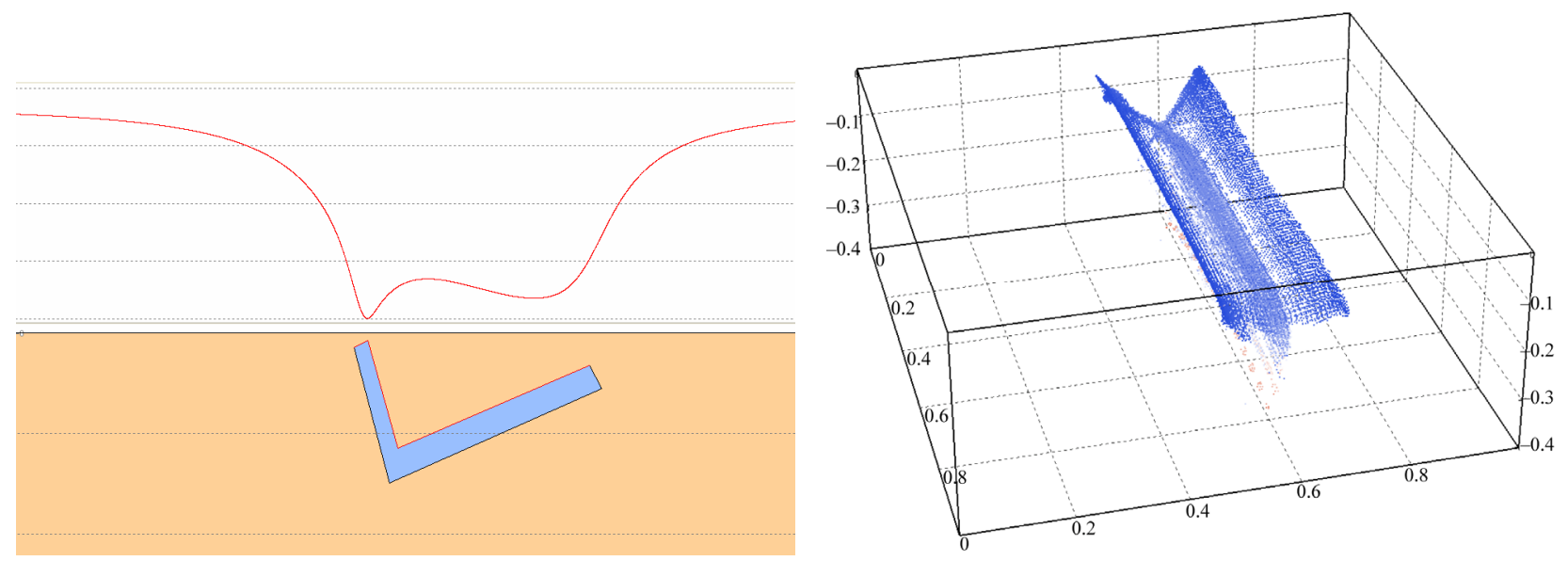

Figure 4. L-rod field shape and the result of its processing. 

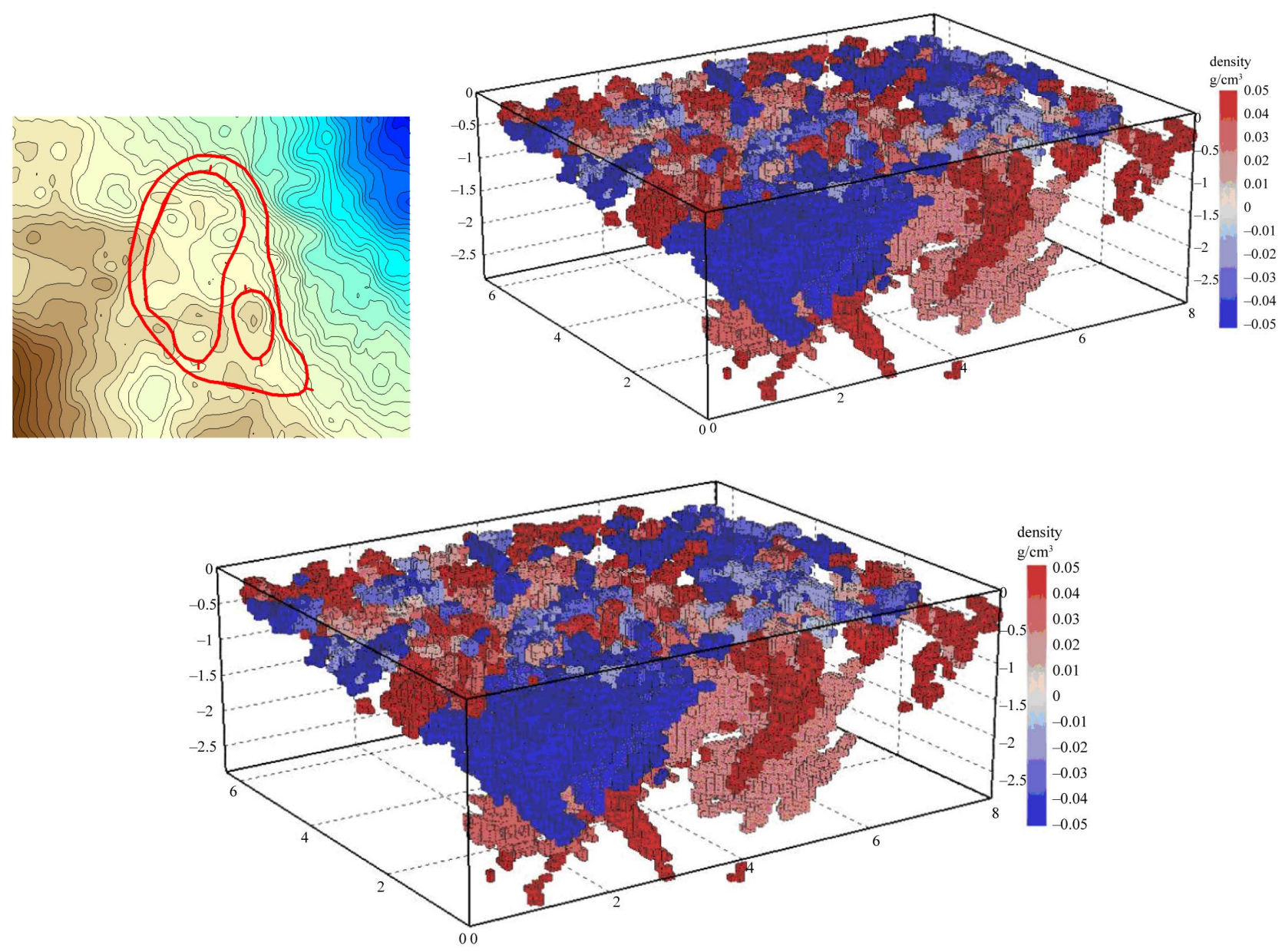

Figure 5. The real field and the result 3D chart. Contour marks the known oil reservoir.

used within quality and quantity interpretation of geophysical surveys.

\section{REFERENCES}

[1] C. Zhang, M. F. Mushayandebvu, A. B. Reid, J. D. Fairhead and M. E. Odegard, "Euler Deconvolution of Gravity Tensor Gradient Data," Geophysics, 2000, Vol. 65, No. 2, pp. 512-520. doi:10.1190/1.1444745

[2] V. O. Mikhailov and M. Diament, "Some Aspects of Interpretation of Tensor Gradiometry Data," Izvestiya, Physics of the Solid Earth, Vol. 42, No. 12, 2006, pp. 971-978. doi:10.1134/S1069351306120019
[3] A. S. Dolgal, "Geopotential Fields Approximation for Practical Tasks Using Equivalent Sources," Geophysical Journal, Vol. 21, No. 4, 1999, pp. 71-80.

[4] A. D. Gvishiani, S. M. Agayan, Sh. R. Bogoutdinov and A. A. Solovyov, "Discrete Mathematical Analysis and Applications Geology and Geophysics," Bulletin of Kamchatka Regional Association "Educational-Scientific Center", Earth Sciences, Vol. 16, No. 2, 2010, pp. 109-125

[5] G. V. Prostolupov and M. V. Tarantin, "Attractive Potential Full Gradient Vectors Transformation," Proceedings of 38th Uspensky Simposium on Theoretical and Practical Aspects of Geologycal Interpretation of Geophysical Fields, Perm, 2011, pp. 245-248 